\title{
BMJ Adverse drug events in a paediatric Open intensive care unit: a prospective cohort
}

\author{
Dafne C B Silva, ${ }^{1}$ Orlei Ribeiro Araujo, ${ }^{2}$ Rodrigo G Arduini, ${ }^{2}$ Carolina F R Alonso, ${ }^{2}$ \\ Audrey R O Shibata, ${ }^{1}$ Eduardo J Troster ${ }^{1}$
}

To cite: Silva DCB,

Araujo OR, Arduini RG, et al. Adverse drug events in a paediatric intensive care unit: a prospective cohort. $B M J$ Open 2013;3:e001868. doi:10.1136/bmjopen-2012001868

- Prepublication history for this paper are available online. To view these files please visit the journal online (http://dx.doi.org/10.1136/ bmjopen-2012-001868)

Received 24 July 2012 Revised 23 January 2013 Accepted 24 January 2013

This final article is available for use under the terms of the Creative Commons Attribution Non-Commercial 2.0 Licence; see http://bmjopen.bmj.com

${ }^{1}$ Faculty of Medicine, Instituto da Criança, University of Sao Paulo, FMUSP, Sao Paulo, Sao Paulo, Brazil

${ }^{2}$ Pediatric Oncology Institute (GRAACC/IOP), Federal University of Sao Paulo, Sao Paulo, Sao Paulo, Brazil

Correspondence to Dr Orlei Ribeiro de Araujo; orlei@uol.com.br

\section{ABSTRACT}

Objectives: To describe adverse drug events (ADEs) in children under intensive care, identify risk factors and tools that can detect ADEs early, and the impact on length of stay (LOS).

Design: A prospective observational study.

Setting: Paediatric intensive care unit of a tertiary care teaching hospital.

Patients: 239 patients with a mean age of 67.5 months representing 1818 days of hospitalisation in intensive care unit.

Interventions: Active search of charts and electronic patient records using triggers. The statistical analysis involved linear and logistic regression.

Measurements and main results: The average LOS was 7.6 days. There were 110 proven, probable and possible ADEs in 84 patients (35.1\%). We observed 138 instances of triggers. The major classes of drugs associated with events were: antibiotics $(n=41)$, diuretics $(n=24)$, antiseizures $(n=23)$, sedatives and analgesics $(n=17)$ and steroids $(n=18)$. The number of drugs administered was most related to the occurrence of ADEs and also to the LOS $(p<0.001)$. The occurrence of an ADE may result in an increase in the LOS by 1.5 days per event, but this was not statistically significant in this sample. Patients aged less than 48 months also proved to be at a significant risk for ADEs, with an OR of $1.84(95 \% \mathrm{Cl} 1.07$ to 3.15 , $p=0.025)$. The number of drugs administered also correlated with the number of ADEs $(p<0.0001)$. The chance of having at least one ADE increased linearly as the patient was administered more drugs.

Conclusions: The use of multiple drugs as well as lower patient age favours the occurrence of ADEs. The active search described here provides a systematic approach to this problem.

\section{INTRODUCTION}

The use of medication in children and infants is a matter of great concern largely owing to the vulnerability of their constantly changing and developing homeostasis, as well as the unique mechanisms by which different children respond to injuries. There are important differences in absorption, distribution,

\section{ARTICLE SUMMARY}

Article focus

- Children have increased susceptibility to drug-related adverse events.

- In intensive care units, multiple, potentially hazardous drugs are routinely administered.

- Active search focused on specific circumstances associated with adverse drug events (ADEs) in specific elements of the patient's chart can increase the rates of observed ADEs.

\section{Key messages}

- The use of multiple drugs as well as lower patient age favours the occurrence of ADEs.

- Active search can provide a systematic approach to identify ADEs in paediatric ICUs (PICUs).

- Definition of triggers and daily search in the records of patients is a simple way to perform an active search for ADEs.

Strengths and limitations of this study

- (-) Our sample did not have the power to implicate ADEs as a causative factor in prolonging PICU stays.

- (-) Time of observation was short, and did not include the seasonality of respiratory diseases.

- (+) We analysed a PICU population in a country outside Europe and North America, in a developing country (Brazil), where ADEs' notification is incipient.

- (+) The defined triggers can be useful in other studies involving ADEs in children.

metabolism and excretion of drugs during childhood and early adolescence. ${ }^{1}$ In addition, safety of several medications has not been properly evaluated in the paediatric age group, while others are prescribed differently from those recommended for adults; key differences include dose and frequency of administration, drug formulation, route of administration or indication for use in childhood (ie, 'off-label' use), and each of these factors can vary depending on the age of the child. ${ }^{2}$ Most of the times, recommended doses of drugs used in children are based on extrapolations from adult doses, related only to weight, body surface area and age, often 
ignoring their pharmacokinetic and pharmacodynamic properties; this results in increased susceptibility of children to drug-related adverse events. ${ }^{1-4}$

Reports documenting the incidence of adverse drug events in the paediatric population range from 4.3 to $16.7 \%$; $12.2 \%$ of these events being serious in nature, with high morbidity and mortality. ${ }^{5}$ Hospitalised children may be at a higher risk of an adverse event, as doses, drug safety and effectiveness are often difficult to determine. ${ }^{6}$ Kaushal $e t a l^{4}$ identified that the potential frequency of ADEs in children is three times higher than a previous study focused on ADEs in adults, however, the rate of avoidable ADEs was similar. In intensive care units, multiple, potentially hazardous drugs are routinely administered, such as inotropes, sedation medications, analgesia and antibiotics; as the risk of an ADE increases by $1.7 \%$ for each additional drug used, ${ }^{5}$ it is far more likely that adverse reactions will occur in the ICU.

There are a few studies documenting safety in drug administration in children in the ICU. The primary objective of this study is to describe ADEs in children admitted to the paediatric ICU (PICU) of a tertiary care hospital in Sao Paulo, Brazil. As secondary objectives, we attempted to identify risk factors for such events and tools that could detect them early as well as determine if there was impact on LOS.

\section{MATERIALS AND METHODS}

We conducted a preliminary survey over a period of 22 days in March 2004 to identify the number of admissions required to effectively report ADEs in the PICU, a unit of 13 beds, with an average occupation of $80 \%$. Based on the results of this survey, we determined that it would then be necessary to study 150 admissions to reach a stable estimate of the incidence of ADEs and explore possible risk factors using a multivariate analysis (approximately 10 ADEs for each variable potentially associated). The study population consisted of consecutive admissions to the PICU between 1 October 2005 and 31 March 2006. The strategy to identify ADEs was through an active search, using pre-established parameters ('triggers'). A 'trigger' can be defined as an occurrence, prompt or flag, found when reviewing a patient's medical chart, that requires further investigations to determine the occurrence or non-occurrence of an adverse event. ${ }^{78}$ Using this method, specific events, such as prescription or abrupt discontinuation of certain medications, prescription of antidotes, and some laboratory tests, serve as indicators for further investigation. Several triggers have been described in the literature, ${ }^{8}$ and therefore we chose and adapted the ones that seemed more consistent with the drugs commonly used in our PICU. Table 1 shows these triggers and the rationale for their use. The positive predictive value (PPV) of each trigger was calculated as the number of times that each trigger identified an ADE, divided by the total number of times the triggers were identified in the active search.

The following methodology was undertaken for an active research:

1. The admission form for each new patient in the PICU was entered by two trained intensive care paediatricians; data were analysed and consolidated by two authors (DCBS and AROS).

2. The following records for each patient were reviewed, guided by triggers indicative of adverse events:

- Laboratory tests (electronic database)

- Clinical annotations

- Nursing annotations

- Prescription.

3. The Naranjo algorithm was applied to classify the cause of the ADE: proven, probable, possible or doubtful. ${ }^{9}$

4. Analysis of all proven, probable and possible ADEs.

The study included only unavoidable ADEs, that is, only those that occurred during normal use of a drug, and not the result of a human error, ${ }^{10}$ as well as those classified as moderate to severe according to the WHO guidelines. By this definition (WHO), ADE is any detrimental or undesirable event, unintended, which appears after administration of a drug at doses normally used for prophylaxis, diagnosis or treatment of a disease. A moderate reaction is one that requires modification of therapy and may require specific treatment; a severe reaction is potentially fatal and requires specific treatment, requires or prolongs hospitalisation. ${ }^{11}$ We analysed only those ADEs that appeared after admission. ADEs that appeared after admission but were related to drugs that the patient was receiving before being admitted were defined as owing to 'prevalent drug'. This definition eliminated, for example, ADEs owing to chemotherapy already present before admission in patients with cancer. ADEs related to drugs introduced after admission were classified as owing to 'incident drug'. The study protocol was reviewed and approved by the Ethics Committee of the institution prior to the start of data collection (protocol number $485 / 56 / 2005)$. Because of the observational nature of the study, without any interference in therapy, informed consent was waived.

Statistical analysis was performed using SPSS and Microsoft Excel, obtaining the OR by logistic regression. A multinomial logistic regression model was performed with the variables 'Presence of chronic disease', 'Age', 'Gender', 'Number of drugs' (independent) and 'ADEs' (dependent). We chose the variables 'Age' and 'number of drugs' because they have been significantly correlated with the incidence of ADEs. ${ }^{5}$ Some studies have shown a lower risk for ADEs in male children. ${ }^{12}$ Chronic illness is an important variable owing to the continuous use of various drugs and the occurrence of organ dysfunction. We also used a linear regression model for the variables 'ADEs', 'Presence of chronic disease', 'Age', 'Gender', 'Number of drugs' (independent) and 'LOS' (dependent). Significance of differences between means was 
Table 1 Rationale for the use of triggers

\begin{tabular}{|c|c|}
\hline Triggers & Rationales for use \\
\hline Haematological alterations & Anemia, leucopoenia, and thrombocytopoenia are adverse reactions of various drugs \\
\hline Biochemical alterations & $\begin{array}{l}\text { Hyponatraemia, hypokalaemia, elevated BUN and creatinine are common events with } \\
\text { various drugs }\end{array}$ \\
\hline Cardiac alterations & $\begin{array}{l}\text { Tachycardia is common, for example, with } \beta \text {-adrenergic agents, which can cause other } \\
\text { arrhythmias; bradycardia may occur with } \beta \text {-blockers }\end{array}$ \\
\hline Antihistamines & Indicator of allergic reaction \\
\hline Corticoids & Potential indicator of allergic reaction \\
\hline Allergic reactions & Frequently reported adverse events \\
\hline $\begin{array}{l}\text { Non-programmed endotracheal } \\
\text { intubation }\end{array}$ & Potential indicator of respiratory depression, common, for example, with benzodiazepines \\
\hline $\begin{array}{l}\text { Level of consciousness } \\
\text { degradations }\end{array}$ & Common with benzodiazepines, anticonvulsants \\
\hline Drug interactions & $\begin{array}{l}\text { For example, hypotension and lethargy caused by concomitant administration of sedatives } \\
\text { and anticonvulsants }\end{array}$ \\
\hline Antiseizures prescription & $\begin{array}{l}\text { Potential indicative of unexpected seizure, when using medications that may lead to } \\
\text { changes in electrolytes and seizures, like amphotericin B }\end{array}$ \\
\hline Drug intolerance & $\begin{array}{l}\text { For example, vomiting and diarrhoea, frequent events with various medications, such as } \\
\text { antibiotics }\end{array}$ \\
\hline $\begin{array}{l}\text { Non-programmed suspension of } \\
\text { drug }\end{array}$ & Indicative of intolerance or adverse reaction \\
\hline Fever & Adverse event of drugs such as amphotericin B \\
\hline Sudden death & Already reported with drug combinations containing dipyrone \\
\hline Serum level alteration & $\begin{array}{l}\text { for monitored drugs such as vancomycin and phenobarbital, with a narrow therapeutic } \\
\text { range and potentially toxic at high levels }\end{array}$ \\
\hline $\begin{array}{l}\text { Aminophylline/adrenaline } \\
\text { prescription }\end{array}$ & Potential indicators of severe allergic reactions \\
\hline Antidotes prescription & $\begin{array}{l}\text { For example, the use of flumazenil may indicate adverse events due to the use of } \\
\text { benzodiazepines }\end{array}$ \\
\hline Others & $\begin{array}{l}\text { Adverse events discovered in the review of medical records, and that does not fit in any } \\
\text { trigger, being the trigger the event itself }\end{array}$ \\
\hline
\end{tabular}

obtained by the t test. Variables involving time were analysed using the Kaplan-Meier method, and $\mathrm{p}<0.05$ was considered statistically significant.

\section{RESULTS}

In our pilot survey, we identified 20 adverse events of moderate-to-severe intensity over a period of 22 days in March 2004, which we calculated to represent at least 5 events/100 patient-days.

In our actual study, there were 244 admissions to the PICU during the period between 1 October 2005 and 31 March 2006. Four patients were excluded because they were adult living-donors for liver transplant, and one patient was excluded owing to age $>18$ years. We analysed the remaining 239 patients, representing a total of 1818 days of PICU hospitalisation. The average length of stay was 7.6 days with a SD of 9.5 days.

The mean age was 67.5 months (median 51 months, range 1-243), and 113 patients (47.2\%) were younger than 48 months. Ninety-four of the 239 patients were male $(39.3 \%)$. Only 39 of the 239 patients did not have a chronic disease at admission $(16.3 \%)$; the most prevalent chronic diseases were cancer $(n=48,20 \%)$, hepatic disease $(n=37,15.4 \%)$, neurological disease $(n=28$, $11.7 \%)$, respiratory disease $(n=28,11.7 \%)$ and cardiac disease $(n=12,5 \%)$. Admissions were mostly owing to respiratory failure $(n=83)$, postoperative of neurosurgical, general or cardiac surgery $(n=52)$, decreased level of consciousness $(n=14)$ or sepsis/septic shock $(n=28)$. Other causes were seizures, digestive bleeding, dehydration, renal failure, hypertension and others.

We observed 138 occurrences of triggers, as shown in table 2, with their predictive positive values. These triggers led to the identification of 110 proven, probable or possible ADEs in 84 patients $(35.1 \%)$ during the 6- month study period, resulting in a rate of 60.5 ADEs/ 1000 patient-days; 21 patients had more than one ADE. Thirty-nine ADEs were owing to prevalent drugs and the remaining $71(64.5 \%)$ were related to drugs introduced after PICU admission or 'incidents' (table 3). Table 4 exhibits the observed ADEs and related drugs.

Gender, the presence of chronic disease, age and administration of at least five drugs were included in a multinomial logistic regression analysis as independent variables for the incidence of ADEs (dependent variable); for males, the OR was 1.31 ( $\mathrm{p}=0.33)$; for the presence of a chronic disease, the OR was $0.71(p=0.35)$ and 
Table 2 Occurrences of triggers used for active search

\begin{tabular}{|c|c|c|}
\hline Triggers & $\begin{array}{l}\text { Number of } \\
\text { occurrences }\end{array}$ & $\begin{array}{l}\text { Positive } \\
\text { predictive } \\
\text { values (\%) }\end{array}$ \\
\hline $\begin{array}{l}\text { Haematological } \\
\text { alterations }\end{array}$ & 8 & 5.79 \\
\hline $\begin{array}{l}\text { Biochemical } \\
\text { alterations }\end{array}$ & 64 & 46.37 \\
\hline Cardiac alterations & 17 & 12.3 \\
\hline Antihistamines & 5 & 3.62 \\
\hline Corticoids & 2 & 1.45 \\
\hline Allergic reactions & 11 & 7.97 \\
\hline $\begin{array}{l}\text { Non-programmed } \\
\text { endotracheal } \\
\text { intubation }\end{array}$ & 1 & 0.72 \\
\hline $\begin{array}{l}\text { Level of } \\
\text { consciousness } \\
\text { degradations }\end{array}$ & 2 & 1.45 \\
\hline Drug interactions & 8 & 5.80 \\
\hline $\begin{array}{l}\text { Antiseizures } \\
\text { prescription }\end{array}$ & 2 & 1.45 \\
\hline Drug intolerance & 0 & 0 \\
\hline $\begin{array}{l}\text { Non-programmed } \\
\text { suspension of drug }\end{array}$ & 1 & 0.72 \\
\hline Fever & 0 & 0 \\
\hline Sudden death & 0 & 0 \\
\hline Serum level alteration & 0 & 0 \\
\hline $\begin{array}{l}\text { Aminophylline/ } \\
\text { adrenaline prescription }\end{array}$ & 0 & 0 \\
\hline Antidotes prescription & 3 & 2.17 \\
\hline Others & 14 & 10.14 \\
\hline
\end{tabular}

none of the individual chronic conditions displayed an increased risk for ADEs; however, patients aged less than 48 months proved to be a significant risk factor, with an OR of 2.1 (95\% CI 1.19 to $3.72, \mathrm{p}=0.01$ ). There was a positive interaction in patients aged less than 48 months and concomitant administration of at least five drugs $(\mathrm{OR}=2.05,95 \%$ CI 1.18 to $3.57, \mathrm{p}=0.01)$ in the occurrence of at least one ADE. This interaction remained significant, with discrete elevation of the OR until nine concomitant drugs were administered $(\mathrm{OR}=2.03,95 \%$ CI 1.15to 3.60, $\mathrm{p}=0.014$, for age $<48$ months; and $\mathrm{OR}=4.69,95 \%$ CI 2.41 to $9.15, \mathrm{p}<0.0001$ for the use of 9 drugs).

In a bivariate analysis, the number of drugs received by each patient correlated with the number of ADEs

Table 3 Distribution of prevalent-drug and incident-drug adverse events according to causality

\begin{tabular}{lccr}
\hline ADE & Prevalent drug & Incident drug & Total \\
\hline Proven & 0 & 5 & 5 \\
Probable & 12 & 32 & 44 \\
Possible & 27 & 34 & 61 \\
Total & 39 & 71 & 110 \\
\hline ADE, adverse drug events. & &
\end{tabular}

$\left(\mathrm{R}^{2}=0.13, \mathrm{p}<0.0001\right)$. The likelihood of at least one ADE became significant when the patient was given at least five drugs at the same time (OR 2.19, 95\% CI 1.14 to $4.20, \mathrm{p}=0.018$ ). We observed a linear elevation of the chance of an occurrence of an ADE as the patient was administered more medications, achieving an OR of 7.26 (95\% CI 2.77 to $19.1, \mathrm{p}<0.0001)$ with 11 concomitant drugs. The same was observed for the occurrence of more than one ADE (table 5).

There was a significant difference between the mean LOS between patients with and without ADEs (11.1 vs 5.3 days, $\mathrm{p}<0.0001)$. In a bivariate linear regression model (LOS as the dependent variable and ADEs as independent), the slope coefficient was 2.75 ( $\mathrm{p}=0.001$ ), meaning that each ADE corresponded to an increase of 2.75 days in the LOS. However, this increase was not maintained when other confounding variables were added in the multivariate regression model. The only independent variables remaining in the final model that affected LOS were the number of ADEs ( $\mathrm{p}=0.089$; slope coefficient 1.49) and the number of drugs $(\mathrm{p}<0.001$; slope coefficient $\left.0.83 ; \mathrm{R}^{2}=0.104\right)$. The slope coefficient could indicate an increase in LOS of 1.49 days for each $\mathrm{ADE}$, if statistically significant, but the study did not have the power to demonstrate it. A sample calculation showed that in order for this fact to be significant in a larger sample, it would take 1000 patients to achieve $\mathrm{p}<0.05$ with a power of 0.94 , considering the observed SD of 9.5 for the dependent variable 'LOS' and 0.72 for independent 'number of ADEs'. Other variables did not show any significant relationship.

In addition, mean 'survival' without ADEs (time from admittance to the PICU until the occurrence of an ADE) was 19 days for patients older than 48 months and 11.2 days for patients younger than 48 months $(\mathrm{p}=0.017)$.

The drug classes involved in ADEs were: antibiotics $(n=41)$, diuretics (24), antiseizures (23), sedatives and analgesics (17), steroids (18), antihypertensives (9), bronchodilators (8), gastric protectors (3), immunosuppressives (4), vasoactive drugs (5), hormonal analogues (4), antipyretics (4) and others (5).

\section{DISCUSSION}

Determining the occurrence of adverse events in an intensive care environment is a complex task. The symptoms of the event may overlap the underlying disease and may be caused by several unrelated factors including the pharmacokinetic profile of the drug, unknown drug allergies of each patient or human error. These difficulties may serve as an explanation for why many events are not recognised as ADEs. Frequently, other drugs are administered in an attempt to solve the problem created by the ADE, without any specific diagnosis. While some events are easily attributed to certain drugs, there are several possible and poorly documented events and some are completely unknown and rare. There are also 
Table 4 Observed adverse drug events (ADEs) and related drugs

\begin{tabular}{|c|c|c|}
\hline ADEs & $\mathbf{N}$ & Related drugs \\
\hline Hyponatremia & 27 & $\begin{array}{l}\text { Furosemide, fentanyl, carbamazepine, vigabatrin topiramate, hydrochlorothiazide, } \\
\text { somatostatin, vancomycin, rifampicin, ranitidine, phenytoin }\end{array}$ \\
\hline Hyperglycemia & 17 & Dexamethasone, hydrocortisone, methylprednisolone, terbutaline, tacrolimus \\
\hline Hypokalaemia & 13 & Amphotericin B, terbutaline, furosemide, ranitidine \\
\hline Skin rash and urticaria & 11 & Vancomycin, dipyrone, cefepime, ceftriaxone, levetiracetam, dipyrone, rasburicase \\
\hline $\begin{array}{l}\text { Hypoventilation/desaturation of } \\
\text { oxygen }\end{array}$ & 6 & Midazolam, propofol, fentanyl, morphine, diazepam \\
\hline Bradycardia & 4 & Midazolam \\
\hline Hypotension & 4 & Midazolam, furosemide, thiopental, chlorpromazine \\
\hline Liver enzyme abnormalities & 4 & $\begin{array}{l}\text { Meropenem, carbamazepine, amlodipine, carvedilol, clonidine, amitriptyline, } \\
\text { phenobarbital }\end{array}$ \\
\hline Hypertension & 3 & Prednisone tacrolimus, dopamine \\
\hline Increased BUN and creatine & 3 & Vancomycin, tacrolimus \\
\hline Seizure & 2 & Hydrocortisone, liposomal amphotericin B, cefepime \\
\hline Tachycardia & 2 & Terbutaline \\
\hline Anemia & 2 & Ketoprofen, paracetamol \\
\hline Extrasystole & 2 & Carvedilol, terbutaline \\
\hline Increased number of platelets & 2 & Meropenem, ceftriaxone \\
\hline Vomiting & 2 & Nitroprusside, tacrolimus \\
\hline Cardiorespiratory arrest & 1 & Dipyrone \\
\hline Thrombocytopenia & 1 & Dipyrone \\
\hline Apnea & 1 & Phenytoin \\
\hline Leucopoenia & 1 & Imipenem \\
\hline Stevens-Johnson syndrome & 1 & Trimethoprim/sulfamethoxazole \\
\hline Eosinophilia & 1 & Ceftriaxone \\
\hline
\end{tabular}

a multitude of patient-specific risk-factors leading to the occurrence of an ADE, including age and certain comorbidities, such as the presence of renal or hepatic impairment. Drug-related factors such as toxicity, time of administration, dosage and duration of use are variables that can also impact the probability of ADEs. In addition, new drugs that have just completed phase III clinical trials may not have been powered to detect rare events. ${ }^{13}$ In general, if we do not look for ADEs, it is unlikely that we will find them. ${ }^{14}$

In the absence of reliable empirical methods for detection of ADEs, formal and logical tools can help differentiate an ADE from a symptom caused by exacerbations in a patient's underlying condition. The most widely accepted formal instrument to obtain this is the
Naranjo algorithm; however, this tool is also not without bias: in our sample, only 5 of 110 ADEs were classified as 'proven' or definite (scores 9 and 10). Therefore, some included events may not have been ADEs. However, to prove an $\mathrm{ADE}$ according to the algorithm, it is necessary to re-administer the drug and observe the event again, or obtain serum levels that are known to be toxic. The first option is strongly discouraged and the second may be technically impossible or unavailable. However, we excluded the 'doubtful' events (score equal to or less than 1) from our analysis and some of these events may have actually been ADEs.

Most of our patients had chronic diseases, which may contribute to a higher incidence of ADEs, owing to the use of multiple medications. This population of chronic

Table 5 ORs related to the concomitant use of medications

\begin{tabular}{|c|c|c|c|c|c|c|}
\hline \multirow[b]{2}{*}{ Number of drugs } & \multicolumn{3}{|c|}{ Occurrence of at least one ADE } & \multicolumn{3}{|c|}{ Occurrence of more than one ADE } \\
\hline & $\overline{\text { OR }}$ & $95 \% \mathrm{Cl}$ & p Value & $\overline{\text { OR }}$ & $95 \% \mathrm{Cl}$ & p Value \\
\hline 5 & 2.19 & 1.14 to 4.2 & 0.018 & 2.38 & 0.67 to 8.38 & 0.175 \\
\hline 6 & 3.03 & 1.69 to 5.40 & 0.0002 & 3.28 & 1.06 to 10.07 & 0.037 \\
\hline 7 & 3.69 & 2.11 to 6.46 & $<0.0001$ & 2.95 & 1.14 to 7.60 & 0.025 \\
\hline 8 & 3.84 & 2.24 to 6.80 & $<0.0001$ & 3.35 & 1.34 to 8.35 & 0.009 \\
\hline 9 & 4.40 & 2.29 to 8.45 & $<0.0001$ & 3.14 & 1.24 to 7.90 & 0.015 \\
\hline 10 & 6.48 & 2.85 to 14.77 & $<0.0001$ & 3.69 & 1.36 to 9.99 & 0.010 \\
\hline 11 & 7.26 & 2.77 to 19.01 & $<0.0001$ & 5.55 & 1.98 to 15.52 & 0.001 \\
\hline
\end{tabular}


patients reflects the current reality of Brazilian university hospitals. We observed no significant difference in ADE incidence between patients with and without chronic diseases, which can be explained by the exclusion of events prior to PICU admission and was more likely related to the medications used regularly.

The incidence of observed events $(35.1 \%$ of admissions) is much higher than those reported in adult patients hospitalised in ICUs (around 9\%). ${ }^{15}$ Furthermore, we found that younger children under the age of 48 months, which constitute approximately half the patient sample, were more likely to have ADEs. This was particularly significant with the administration of over five drugs at the same time, and also resulted in an ADE earlier in the patient's ICU stay. The high incidence of ADEs also highlights the importance of an active search focused on triggers to detect ADEs in children: Holdsworth et al reported ADE rates of 6/100 admissions (ICU and paediatric ward, determined using a chart review by a pharmacist).${ }^{16}$ Takata et al performed a search focused on triggers and reported 11.1 events/ 100 inpatients, almost double that of the retrospective study. These authors indicated that performing a search focused on specific circumstances associated with ADEs in specific elements of the patient's chart can increase the rates of observed ADEs. ${ }^{17}$ The methodology used in this study (definition of triggers and daily search in the records of patients) is a simple way to perform an active search for ADEs. Triggers can be individualised for each hospital setting according to the most frequently used medications. PPVs can be determined through a simple calculation that assists in the choice of triggers that are most useful in each unit. We observed higher PPVs for biochemical alterations; in an automated process, the system of the laboratory itself could alert for possible ADEs.

We wish to highlight that our study evaluated severely ill children under intensive care receiving multiple drugs (up to 18), and the chances of developing an $\mathrm{ADE}$ is therefore, more likely. In addition, comparing event rates is also challenging and potentially misleading as definitions of ADEs are unclear among studies, ranging from a benign and transient alteration of electrolytes to vital organ damage. ${ }^{10}$

We investigated whether ADEs may have an impact on the LOS in the PICU. The most important limitation of the study was that our sample did not have the power to implicate ADEs as a causative factor in prolonging PICU stays by 1.5 days; however, calculations based on SD observed in our sample showed a high probability that this would be true in a longer term study. In addition to possible patient harm, there are significant costs associated with patient stays in the ICU: An increase of 1.5 days/event results in an additional 330 days/year. Estimating cost at US\$600.00 each day, ADEs amount to US $\$ 198000 /$ year, which is a considerable sum for our public health system. In principle, the events occurred as a result of habitual use of drugs and were therefore 'inevitable'; however, a systematic approach could convert some ADEs from presumably inevitable to avoidable. A good example cited by Kane-Gill et $a l^{10}$ describes bleeding caused by the correct dose of heparin in a patient being monitored by partial thromboplastin time and would be classified as inevitable. If an investigation identified that the laboratory has changed their method for thromboplastin time and failed to communicate the necessary adjustment, the error would become preventable. More studies on pharmacokinetics and drug interactions in children are required to define optimal dosing regimens and reduce ADEs.

Another limitation of the study was the short time of observation, which did not include the seasonality of respiratory diseases. A positive aspect of the study was the analysis of a PICU population in a country outside Europe and North America, therefore making it possible to analyse ADEs owing to drugs such as dipyrone. In Brazil, the reporting of ADEs is incipient. The online system provided by the health authority only receives notifications, which are not mandatory. Active search is not utilised, even in private institutions. We hope that our study will contribute to a future systematic approach to this subject in developing countries.

\section{CONCLUSIONS}

The use of multiple drugs as well as lesser patient age favours the occurrence of ADEs, which in turn may result in an increase in the length of PICU hospitalisation. The use of an active search using triggers can provide a systematic approach to identify ADEs in PICUs.

Contributors DCBS was responsible for the design, acquisition of data and article drafting. ORA and RGA were responsible for article drafting, analysis and interpretation of data. AROS and CFRA worked on acquisition of data and contributed to the interpretation of the results. EJT conceived the study and revised the draft. All authors read and approved the final manuscript.

Funding This research received no specific grant from any funding agency in the public, commercial or not-for-profit sectors.

Competing interests None.

Ethics approval Ethics Committee of the institution-Sao Paulo University (USP).

Provenance and peer review Not commissioned; externally peer reviewed.

Data sharing statement No additional data are available.

\section{REFERENCES}

1. Niederhauser VP. Prescribing for children: issues in pediatric pharmacology. Nurse Pract 1997;22:16-18.

2. Hussain $E$, Kao $E$. Medication safety and transfusion errors in the ICU and beyond. Crit Care Clin 2005;21:91-110.

3. Wilson RM, Runciman WB, Gibberd RW, et al. The quality in Australian Health Care Study. Med J Aust 1995;163:458-71.

4. Kaushal R, Bates DW, Landrigan C, et al. Medication errors and adverse drug events in pediatric inpatients. JAMA 2001;285:2114-20.

5. Impicciatore P, Choonara I, Clarkson A, et al. Incidence of adverse drug reactions in paediatric in/out-patients: a systematic review and meta-analysis of prospective studies. Br J Clin Pharmacol 2001;52:77-83.

6. Gill AM, Leach $\mathrm{HJ}$, Hughes J, et al. Adverse drug reactions in a paediatric intensive care unit. Acta Paediatr 1995;84:438-41. 
7. Rozich JD, Haraden CR, Resar RK. Adverse drug event trigger tool: a practical methodology for measuring medication related harm. Qual Saf Health Care 2003;12:194-200.

8. Resar RK, Rozich JD, Classen DC. Methodology and rationale for the measurement of harm with trigger tools. Qual Saf Health Care 2003;12(Suppl 2):ii39-45.

9. Naranjo CA, Busto $U$, Sellers EM, et al. A method for estimating the probability of adverse drug reactions. Clin Pharmacol Ther 30:239-45.

10. Kane-Gill SL, Kirisci L, Verrico MM, et al. Analysis of risk factors for adverse drug events in critically ill patients. Crit Care Med 2012;40:823-8.

11. World Health Organization (WHO). Council for International Organizations of Medical Sciences. Guidelines for preparing core clinical safety information on drug from CIOMS Working Group III. Geneva, 1995.
12. Smyth RM, Gargon E, Kirkham J, et al. Adverse drug reactions in children-a systematic review. PLOS ONE 2012;7:e24061.

13. Brown SD Jr, Landry FJ. Recognizing, reporting, and reducing adverse drug reactions. South Med J 2001;94:370-3.

14. Kelly WN. How can I recognize an adverse drug event. http://www. medscape.org/viewarticle/569794 (accessed 1 Feb 2012)

15. Vargas E, Terleira A, Hernando F, et al. Effect of adverse drug reactions on length of stay in surgical intensive care units. Crit Care Med 2003;31:694-8.

16. Holdsworth MT, Fichtl RE, Behta M, et al. Incidence and impact of adverse drug events in pediatric inpatients. Arch Pediatr Adolesc Med 2003;157:60-5.

17. Takata GS, Mason W, Taketomo C, et al. Development, testing, and findings of a pediatric-focused trigger tool to identify medication-related harm in US children's hospitals. Pediatrics 2008;121:e927-3 